\title{
Evaluation of the Health Education Impact Questionnaire (heiQ), a Self-Management Skill Assessment Tool, in Italian Chronic Patients
}

This article was published in the following Dove Press journal: Psychology Research and Behavior Management

\author{
Andrea Pozza (1) \\ Richard H Osborne (iD) ${ }^{2,3}$ \\ Gerald R Elsworth (iD ${ }^{2}$ \\ Giacomo Gualtieri $^{4}$ \\ Fabio Ferretti (iD) \\ Anna Coluccial \\ 'Department of Medical Sciences, \\ Surgery and Neurosciences, University of \\ Siena, Siena, Italy; ${ }^{2}$ Centre for Global \\ Health and Equity, Swinburne University \\ of Technology, Melbourne, Australia; \\ ${ }^{3}$ Department of Public Health, University \\ of Copenhagen, Copenhagen, Denmark; \\ ${ }^{4}$ Legal Medicine Unit, Santa Maria alle \\ Scotte University Hospital, Siena, Siena, \\ Italy
}

Background: The Health Education Impact Questionnaire (heiQ) aims to evaluate eight self-management skills in people with chronic conditions. Knowledge about the relations between these self-management skills and different quality of life (QoL) outcomes has received little attention. It is also important to provide further evidence on its properties in non-English healthcare contexts, as the questionnaire is being used in cross-cultural research. Furthermore, in the Italian healthcare context, the relationship between the medical staff and the patients remains asymmetrical, with the latter having the role of passive recipients of medical prescriptions and services. The current study provided further evidence about the psychometric properties of the heiQ among Italian people with chronic conditions, specifically by assessing the factor structure, reliability, convergent/divergent and criterion validity (ie, the specific contribution of each of the self-management skills to QoL outcomes).

Methods: Two hundred ninety-nine individuals with a chronic condition (mean age $=61.4$ years, 50\% females) completed the heiQ and the Medical Outcomes Study-Short Form (MOS SF-36). Confirmatory factor analyses, Composite Reliability Indices (CRI), bivariate correlations and linear regression analyses were computed.

Results: A model with 8 correlated factors showed good fit, in a similar way to previous studies. CRI values were acceptable to good for all the subscales. Associations between some of the heiQ subscales and some of the MOS SF-36 subscales supported criterion validity. In particular, it was confirmed by the moderate associations between the constructive attitudes and approaches subscale and the MOS SF-36 vitality and perceived mental health and by the moderate correlations between the health directed activities subscale and the MOS SF-36 Vitality. In linear regressions, higher emotional distress predicted higher physical and mental QoL, while higher mental QoL was also associated with stronger constructive attitudes and approaches.

Conclusion: The heiQ has robust properties across translations and it can also be used routinely in Italian healthcare contexts. The evidence that all the other self-management skills did not predict either physical or mental QoL could suggest that the self-management model of chronic diseases is still not sufficiently developed in the Italian context, where patients are passive recipients of medical instructions.

Keywords: chronic diseases, self-management, health education, psychometric properties, health status, quality of life, diabetes, cardiovascular diseases

\section{Background}

Chronic diseases represent the leading source of death and disability and account for the majority of the healthcare costs in the industrialized world. ${ }^{1-4}$ In the 2010 report on the global status of the challenges presented by chronic diseases, the World Health
Department of Medical Sciences, Surgery and Neurosciences, University of Siena,

Viale Bracci 16, Siena 53100, Italy

Tel +390577586409

Fax +390577233222

Email ferrefa@unisi.it 
Organization highlighted that non-communicable diseases including chronic conditions such as cardiovascular diseases, diabetes, and chronic respiratory diseases - accounted for nearly two-thirds of deaths worldwide. ${ }^{5}$ Therefore, the increasing number of people with chronic diseases is a challenge for health care systems that requires a radical change in the health services paradigms. This change must include more active involvement of patients in the care process, the self-management of their disease(s), and greater attention to the quality of life outcomes of those affected. Consequently, well-developed, robust and reliable tools are essential to enable the detection of key variables implicated in the improvement of these outcomes. ${ }^{6-8}$

In Australia, Osborne and colleagues developed the Health Education Impact Questionnaire (heiQ), ${ }^{9}$ a self-report questionnaire, which assesses eight independent dimensions related to key self-management skills for people with chronic conditions. These dimensions include Positive and active engagement in life, Health-directed activities, Technique acquisition, Constructive attitudes and approaches, Selfmonitoring and insight, Health services navigation, Social integration and support, and Emotional distress. The measure was originally developed to detect the benefits of health education programs for a wide range of patient groups and specifically sought to provide useful information to medical staff, researchers and policymakers. The items, which were developed from the patients' perspective and assessed with a Likert response format, have been well-tested and found to be robust using a range of psychometric methods. ${ }^{9,10}$ Consistent research has demonstrated that the heiQ is able to detect the improvement in self-management skills in individuals diagnosed with chronic diseases undergoing health education programs in outpatient and community care settings. Since its development, the heiQ has undergone minor refinements. The original version of the questionnaire had 42 items with a 6-point response format. Analyses during the construction of the questionnaire suggested that some items had disordered thresholds: some respondents were unable to differentiate between the two midpoints "slightly agree" and "slightly disagree". Further analyses also suggested that two items could be removed without compromising content validity. Thus, the response format was simplified to a 4-point scale ("strongly disagree" to "strongly agree") and 2 items were removed. 9

Since concepts related to health can be hypothesized to depend on socio-cultural aspects, the heiQ has been translated and adopted in different healthcare and socio-cultural contexts, including Canada, Germany, France, the Netherlands, Norway, and Japan. ${ }^{11-15}$ Schuler and colleagues ${ }^{14}$ tested the factor structure in a large group of German patients with different types of chronic diseases evaluating separately the fit of each of factors. Using highly restrictive confirmatory factor analysis, the hypothesized models of the dimensions Positive and active engagement in life, Constructive attitudes and approaches, and Skill and technique acquisition had good fit. In contrast, the remaining five factors showed inadequate fit in at least one index. When freeing an error covariance in respective models, fit indices improved in a way that model fit was acceptable. Estimates of internal consistency of the scales were acceptable. ${ }^{14}$ Moreover, findings regarding convergent and divergent validity with measures of quality of life, depression and anxiety showed that all the heiQ scales had at least low to moderate correlations with such dimensions. These analyses indicated that the heiQ scales with the strongest affective elements had the highest correlations with measures of mental health. ${ }^{14}$

Further validation studies in the other countries and settings, ${ }^{11,12,15}$ conducted through highly restrictive tests such as confirmatory factor analyses, suggested that the questionnaire performs well, the eight scales are distinct, despite it should be noted that the data rarely supported perfect fit. Across the validation studies, some factors showed cross-loadings and some items presented intercorrelated residuals.

Despite the growing research on the validity and reliability of the heiQ in different countries and socio-cultural contexts, there is a lack of tools measuring self-management skills in the Italian healthcare contexts. Furthermore, the relationship between the self-management skills, as assessed by the heiQ and QoL outcomes have received very little attention. The study performed by Schuler and colleagues ${ }^{14}$ was the only one investigating this aspect by measuring QoL through the Medical Outcomes Survey Short Form-36 (MOS SF-36). ${ }^{16}$ Emotional distress, measured by the heiQ, was negatively correlated with all the QoL outcomes assessed by the MOS SF-36 with correlations ranging from -0.34 to -0.62 . On the other hand, Positive and active engagement in life, Constructive attitudes and approaches, and (to a lesser extent) Skill and technique acquisition were moderately and positively correlated only with General health, Social functioning, Vitality and Mental health outcomes (all correlations $>0.29$ ). Self-monitoring and insight, Health service navigation and Health directed activities were, however, generally only weakly associated with Health Status outcomes. Overall, these data could suggest that some skills have a specific association with some specific outcomes of QoL. The study, ${ }^{14}$ however, did not test the associations between the 
self-management skills and the composite indices, measured by the MOS SF-36, which cover physical and mental QoL. In addition, only bivariate correlations were calculated but the specific contribution of each skill was not assessed and compared with all the other skills in a regression model.

\section{Rationale for the Current Study}

There is a growing body of research that has provided overall support for the factor structure and the reliability of the heiQ. However, evidence about the relations between selfmanagement skills measured by the heiQ and QoL outcomes is limited. Further knowledge could improve its usefulness in research and practice on education programs for chronic conditions. Given the role of self-management skills for the improvement of QoL among people with chronic conditions, it is important to explore the potential contribution of such skills on different QoL domains. It is also important to provide further evidence on the properties of the heiQ in nonEnglish healthcare contexts, as the questionnaire is being used in international cross-cultural research. Finally, it is important to note that it is not a questionnaire per se that is validated, rather it is the data and its interpretation in particular contexts, for particular purposes, that is validated. ${ }^{17}$ Consequently, exploration of the psychometric properties of the heiQ in the Italian context is warranted.

Furthermore, given the imperative of instilling selfmanagement into routine healthcare, and the lack of attention Italian practitioners have given to patients' self-management skills in the healthcare pathway to date, patients in Italy and similar countries are failing to keep up with international evidence and the move to more patient-centred healthcare. In many European countries, the relationship between the medical staff and the patients remains asymmetrical, with the latter having the role of passive recipients of medical prescriptions and services. ${ }^{6,18}$ With these considerations, the aims of the current study were to provide further evidence about the psychometric properties of the heiQ among Italian adults with chronic conditions, specifically by assessing the heiQ's factor structure and reliability. In addition, the study aimed to assess convergent and divergent validity by exploring the associations between the self-management skills. In particular, in accordance with previous evidence ${ }^{14}$ we expected to find a) moderate correlations between Positive and active engagement in life, Constructive attitudes and approaches, and Social integration and support, b) moderate associations between Skill and technique acquisition and Health service navigation and c) weak associations between Emotional distress and all the other self-management skills.
Finally, we assessed criterion validity, ie the specific contribution of each of the self-management skills to QoL outcomes.

\section{Methods}

The study included two phases. First, the original heiQ was translated and culturally adapted in Italian, following the standardised protocol of the Deakin University. ${ }^{19}$ In the second phase, the psychometric properties of the measure were investigated in a large group of adults with chronic diseases.

\section{Translation and Cultural Adaptation of the heiQ}

The translation and cultural adaptation process consisted of a first step with a forward translation by a professional, licensed translator as requested in the protocol (ItalianEnglish bilingual, native Italian speaker) who followed an item intent guide, a second phase with a critical review by the Italian research staff (AP, FF, AC), and finally a blinded independent back translation (Italian-English bilingual, native English speaker). Apart from the translation, items were reviewed for cultural appropriateness. The meaning of every statement in the final translation was checked with one of the original authors (RHO) against all previous language versions through written reports and a consensus online meeting.

\section{Participants and Procedure}

Two hundred ninety-nine individuals with a diagnosis of a chronic condition participated. Mean age was 61.4 years old $(\mathrm{SD}=13.4$, range $=23-86), 50 \%$ of the sample were females. Forty-nine percent were adults (23-64-year old participants; age range $=23-64$ years) and $51 \%$ were older adults (participants with age of 65 years or older; age range= 65-86 years). Twenty-six percent had a degree or a highschool license while $74 \%$ had a lower educational level.

The most common chronic conditions were Diabetes (44.50\%), Arthritis (16.70\%) and Hypertension (10.70\%). About five percent presented one or more chronic conditions. Time since the diagnosis, measured in terms of months, was $114.9(\mathrm{SD}=898.9)$.

An overview of demographic and clinical characteristics is presented in Table 1. Participants were recruited in outpatient wards at a University Hospital and at community healthcare outpatient services. Data were collected from October 2013 to May 2014. All the participants completed the questionnaires individually with the assistance of a psychologist, who 
Table I Demographic and Clinical Characteristics of the Participants (N=299)

\begin{tabular}{|c|c|c|c|}
\hline & $\mathrm{n}$ & $\%$ & $\begin{array}{l}\text { M (SD; } \\
\text { Range) }\end{array}$ \\
\hline Female gender & 150 & 50 & \\
\hline Age (years) & & & $\begin{array}{l}61.4(13.4 ; \\
23-86)\end{array}$ \\
\hline $\begin{array}{l}\text { Recruitment centres } \\
\text { Santa Maria alle Scotte University Hospital } \\
\text { of Siena } \\
\text { Azienda USL Toscana Sud Est } \\
\text { S. Giovanni Rotondo Casa Sollievo della } \\
\text { Soffferenza Hospital } \\
\text { Azienda USL Toscana Centro }\end{array}$ & $\begin{array}{l}61 \\
32\end{array}$ & $\begin{array}{l}21 \\
10.7 \\
8.4\end{array}$ & \\
\hline $\begin{array}{l}\text { Diagnosis of chronic diseases } \\
\text { Diabetes } \\
\text { Arthritis } \\
\text { Hypertension } \\
\text { Other chronic diseases } \\
\text { Systemic sclerosis } \\
\text { Kidney diseases } \\
\text { Cardiovascular diseases } \\
\text { Obstructive pulmonary disease chronic } \\
\text { bronchitis } \\
\text { Duration since the diagnosis of chronic } \\
\text { disease (months) } \\
\text { Polypathologies of chronic diseases }\end{array}$ & $\begin{array}{l}133 \\
50 \\
32 \\
27 \\
19 \\
18 \\
14 \\
6\end{array}$ & $\begin{array}{l}44.5 \\
16.7 \\
10.7 \\
9 \\
6.4 \\
6 \\
4.7 \\
2\end{array}$ & $\begin{array}{l}\text { I14.9 (89.9; } \\
\text { I-480) }\end{array}$ \\
\hline
\end{tabular}

provided information about the aims of the study. In accordance with the Ethical Principles of Psychologists and Code of Conduct, ${ }^{20}$ all the participants, who were included, provided written informed consent to participate in the study after having received a detailed description of the aims.

\section{The Health Education Impact Questionnaire (heiQ)}

The heiQ is a self-report measure which covers eight dimensions, each one representing specific self-management capacities and skills: 1 . Positive and active engagement in life, 2. Health directed activities, 3. Skill and technique acquisition, 4. Constructive attitudes and approaches, 5. Self-monitoring and insight, 6. Health service navigation, 7. Social integration and support, and 8. Emotional distress. Each dimension is assessed by between four and six questions, with forty questions overall. Responses are marked on a 4-point Likert scale, using the endpoints "strongly disagree/strongly agree". A higher score indicates stronger skills in the management of the chronic condition, except in the emotional distress dimension, which is negatively scored.

\section{Medical Outcomes Survey Short Form-36 (MOS SF-36)}

The Medical Outcomes Survey Short Form-36 (MOS SF$36)^{16}$ was used as a measure of QoL to explore criterion validity of the heiQ scales. The MOS SF-36 is a generic measure of QoL which assesses eight health-related dimensions: Physical Functioning (10 items), Role Limitations due to Physical Problems (4 items), Bodily Pains (2 items), General Health Perceptions (5 items), Vitality (4 items), Social Functioning (2 items), Role Limitations due to Emotional Problems (2 items), and Perceived Mental Health (5 items). In addition, a single item provides an indication of perceived change in general QoL over a oneyear time period, is included in the MOS SF-36. The scales of the Italian version ${ }^{21}$ have demonstrated good internal consistency with Cronbach's alpha ranging from 0.78 to 0.93 . In the current study, the Cronbach alpha of the MOS SF-36 scales ranged between 0.81 and 0.91 .

\section{Statistical Analysis}

Distributional properties of the heiQ items were evaluated through skewness and kurtosis indices. Subsequently, to examine fit of the data to the factor structure, confirmatory factor analysis (CFA) was performed through structural equation modelling. ${ }^{22}$ Following the factor structure reported in the original validation study, ${ }^{9}$ an a priori model was tested with eight correlated factors. To evaluate goodness of fit of the model, the following indices recommended by $\mathrm{Hu}$ and Bentler $^{23}$ were used: the Goodness of Fit Index (GFI), the Comparative Fit Index (CFI), the Tucker Lewis Index (TLI): values ranging from 0.95 and 1 indicate excellent fit, from 0.90 and 0.95 good fit. In addition, the Root Mean Square Residual (RMR) was considered: values less than 0.08 indicate acceptable fit, and those less than 0.05 good fit. ${ }^{24}$ Reliability was examined as internal consistency through the calculation of composite reliability indices (CRI), in order to compare the results of the Italian heiQ with those reported in previous validation studies on the measure. CRI values can be interpreted like Cronbach alpha. In the current study, CRI values were assessed according to Nunnally and Bernstein's guidelines ${ }^{25}$ (CRI $>0.70=$ acceptable, CRI $>0.80=$ good, CRI $>0.90=$ excellent $)$.

One-way ANOVAs were calculated, in order to compare means on the heiQ total and subscales between male and female subgroups and between adults and older adults. 
Effect sizes were computed as Cohen's $d$ indices and interpreted according to Cohen's following criteria: ${ }^{26} d \geq|.20|=$ small; $d \geq|.50|=$ medium; $d \geq|.80|=$ large. Criterion validity was examined investigating Pearson's bivariate correlations between the heiQ scores and the MOS SF-36 scores, which was used as a measure of QoL. Values on the correlation coefficients were interpreted according to the following criteria: ${ }^{26} 0<\mathrm{r}<|.30|=$ weak; $|.30|<\mathrm{r}<|.50|=$ moderate; $|.50|<\mathrm{r}<|.70|=$ strong; $|.70|<\mathrm{r}<|1|=$ very strong. Fisher' s z coefficients were calculated to compare the magnitude of bivariate correlations between scores on each of the heiQ subscale and those on the MOS SF-36 composite indices. Power calculations were run for this analysis: for a medium effect size, $80 \%$ power, and significance set at the level described above, the required sample size for bivariate correlations was 162 participants.

Subsequently, to test the specific contribution of each selfmanagement skill on physical health and mental QoL separately, a multiple linear regression analysis with a step-wise method was conducted entering the scores on all the heiQ scales as predictors and the scores on the two MOS SF-36 composite indices as dependent variables. Power calculations were run for this analysis: for a medium effect size, $80 \%$ power, and significance set at the level described above, the required sample size for bivariate correlations was 148 participants. The statistical analysis was conducted using the SPSS software version 21.00 and the AMOS software.

\section{Results}

\section{Confirmatory Factor Analysis}

First, a model with eight correlated factors was tested. However, despite values on the $\chi^{2 / \mathrm{df}}$ was in the range between 1 and 3 and values on the RMSEA and the RMR were lower than 0.06 , suggesting good fit, values on the GFI, CFI and TLI were still not acceptable, evidencing partially inadequate fit. Subsequently, to further improve the model fit, modification indices were inspected and covariances between residuals of some items were added. In particular, covariances were added between the residuals of the item 15 ("I feel like I am actively involved in life") loading on 1. Positive and active engagement in life and those of the item 8 ("I am doing interesting things in my life"), loading on the same factor. Covariances were added between the residuals of the item 3 ("As well as seeing my doctor, I regularly monitor changes") and those of the item 17 ("I carefully watch my health and do what is necessary to keep"), which both loaded on 5. Selfmonitoring and insight. Covariances were added also between the residuals of the item 3 and those of the item 37 ("I get enough chances to talk about my health"). Finally, covariances were added between the residuals of the item 34 ("My health problems do not ruin my life") and those of the item 39 ("I do not let my health problems control my life"), which both loaded on 4. Constructive attitudes and approaches. Covariances were added between the residuals of the item 32 ("I confidently give healthcare professionals the information") loading on 6 . Health service navigation and those of the item 16 ("When I have health problems, I have a clear understanding of what I need to do") loading on 5. Self-monitoring and insight. This model with the inclusion of covariances between residuals of some items showed improved fit in all the indices as compared with the previous models. Fit indices of the tested models and the comparisons with previous validation studies are presented in Table 2.

\section{Reliability}

Reliability was assessed by the calculation of CRI values. Findings showed that CRI were acceptable to good for all

Table 2 Fit Indices of the heiQ Models Tested in the Current Study $(\mathrm{N}=299)$ and the Models Tested in Previous Studies

\begin{tabular}{|c|c|c|c|c|c|c|c|}
\hline Models Tested in the Current Study & $\chi^{2}$ & $\chi 2 / d f$ & GFI & CFI & TLI & RMSEA & RMR \\
\hline Correlated factors & II83.74* & 1.75 & 0.83 & 0.88 & 0.87 & 0.05 & 0.05 \\
\hline Correlated factors with covariances between residuals & $1094.28 *$ & 1.63 & 0.85 & 0.91 & 0.90 & 0.04 & 0.05 \\
\hline \multicolumn{8}{|l|}{ Fit indices in previous validation studies } \\
\hline Original Australian study ${ }^{9}$ & $1420 *$ & 1.80 & n.r & 0.99 & n.r. & 0.04 & n.r \\
\hline Dutch study ${ }^{10}$ & $1257.77^{*}$ & 1.76 & n.r & 0.89 & 0.88 & 0.05 & n.r \\
\hline French study'I & $1210.15^{*}$ & 1.69 & n.r & 0.98 & 0.91 & 0.06 & n.r \\
\hline German study ${ }^{13}$ & $2223.96 *$ & 3.32 & n.r & 0.92 & n.r. & 0.04 & n.r \\
\hline
\end{tabular}

Notes: ${ }^{*} \mathrm{p}<.00$ I; n.r, not reported in the study paper.

Abbreviations: CFI, Comparative Fit Index; heiQ, health education impact Questionnaire; GFI, Goodness of Fit Index; RMR, Root Mean Residual; RMSEA, Root Mean Squared Error Residual Approximation; TLI, Tucker-Lewis Index. 
the subscales according to the criteria proposed by Nunnally and Bernstein. ${ }^{25}$ On one hand, values were comparable with those reported in the Australian original study $^{9}$ for the 2 . Health directed activities, the 3. Skill and technique acquisition, the 8. Emotional distress. On the other hand, values were lower (acceptable instead of good) for the 1. Positive and active engagement in life, the 4. Constructive attitudes and approaches, the 6. Health service navigation than the Australian study. ${ }^{9}$ CRI values for all the heiQ subscales and the comparison with previous validation studies are provided in Table 3.

\section{Group Differences on Self-Management Skills}

Significant differences were observed between adults and older adults on the scores of the 3. Skill and technique acquisition, 7. Social integration and support and 6. Health service navigation subscales: adults had significantly higher scores on these subscales than older adults with small to medium effect sizes. No difference was found between the groups on all the other heiQ subscales. No difference was found between males and females on the scores of heiQ subscales (see Tables 4 and 5).

\section{Associations Between Self-Management Skills}

Bivariate correlations between scores on the heiQ subscales are presented in Table 6. Intercorrelations were all moderate between scores on five subscales (1. Positive and active engagement in life, 4. Constructive attitudes and approaches, 5. Self-monitoring and insight, 6. Skill and technique acquisition, 7. Social integration and support). Intercorrelations between scores on the 2 . Health directed activities subscale and the other subscales were moderate only with those on the 1. Positive and active engagement in life subscale. Intercorrelations between scores on the Emotional distress subscale were moderate and negative only with those on the 4. Constructive attitudes and approaches subscale.

\section{Associations Between Self-Management Skills and QoL Dimensions}

Scores on the 1. Positive and active engagement in life, on the 7. Social integration and support, and on the 6. Health service navigation subscales positively and weakly correlated with those on the MOS SF-36 physical health composite index. Scores on the 5. Self-monitoring and insight subscale positively and moderately correlated with those on the MOS SF-36 physical health composite index. Scores on the 8. Emotional distress subscale negatively and moderately correlated with those on the MOS SF-36 physical health composite index.

Scores on the 2. Health directed activities, 5. Selfmonitoring and insight, heiQ 7. Social integration subscales and support positively and weakly correlated with those on the MOS SF-36 mental health composite index.

Scores on the 4. Constructive attitudes and approaches subscale positively and moderately correlated with those on the SF-36 mental health composite index. Scores on the 8. Emotional distress scale negatively and moderately correlated with those on the MOS SF-36 mental health composite index. No significant difference was found in the magnitude of the correlations between each of the heiQ subscale scores and the two MOS SF-36 composite indices, as demonstrated by the values of the Fisher's $\mathrm{z}$ coefficients. Bivariate correlations between scores on the heiQ and those on the MOS SF-36 composite indices are presented in Table 7.

Table 3 Internal Consistency Values of the heiQ Scales in the Current Study (N=299) and in Previous Studies

\begin{tabular}{|l|l|l|l|l|l|}
\hline \multirow{2}{*}{ heiQ Scales } & Current Study & Original Australian Study & Norwegian Study $^{{ }^{14}}$ & French Study $^{{ }^{\prime \prime}}$ & Dutch Study $^{\mathbf{1 0}^{\prime}}$ \\
\cline { 2 - 6 } & CRI & CRI & CRI & CRI & CRI \\
\hline I. Positive and active engagement in life & 0.72 & 0.83 & 0.79 & 0.86 & 0.81 \\
2. Health directed activities & 0.84 & 0.82 & 0.80 & 0.87 & 0.82 \\
3. Skill and technique acquisition & 0.71 & 0.73 & 0.76 & 0.81 & 0.82 \\
4. Constructive attitudes and approaches & 0.76 & 0.83 & 0.83 & 0.81 & 0.86 \\
5. Self-monitoring and insight & 0.65 & 0.70 & 0.61 & 0.69 & 0.67 \\
6. Health service navigation & 0.78 & 0.82 & 0.72 & 0.80 & 0.85 \\
7. Social integration and support & 0.77 & 0.88 & 0.83 & 0.87 & 0.85 \\
8. Emotional distress & 0.86 & 0.88 & 0.90 & 0.87 & 0.89 \\
\hline
\end{tabular}

Abbreviations: CRI, Composite Reliability Indices, heiQ, health education Impact Questionnaire. 
Table 4 Comparisons on the heiQ Scale Scores Across Age Cohorts ( $N=299)$

\begin{tabular}{|c|c|c|c|c|c|c|c|}
\hline \multirow[t]{2}{*}{ heiQ Scales } & \multirow[t]{2}{*}{ Age Cohorts } & \multirow[t]{2}{*}{ Mean } & \multirow[t]{2}{*}{ SD } & \multicolumn{2}{|c|}{ 95\% Confidence Interval } & \multirow[t]{2}{*}{$F_{(1,298)}$} & \multirow[t]{2}{*}{ Cohen's d } \\
\hline & & & & Lower Limit & Upper Limit & & \\
\hline \multirow[t]{2}{*}{ I. Positive and active engagement in life } & Adults & 3.58 & 0.89 & 3.35 & 3.80 & 1.37 & -.21 \\
\hline & Older adults & 3.41 & 0.70 & 3.24 & 3.58 & & \\
\hline \multirow[t]{2}{*}{ 2. Health directed activities } & Adults & 2.46 & 0.79 & 2.26 & 2.66 & 1.74 & 0.01 \\
\hline & Older adults & 2.65 & 0.82 & 2.44 & 2.85 & & \\
\hline \multirow[t]{2}{*}{ 3. Skill and technique acquisition } & Adults & 2.98 & 0.58 & 2.83 & 3.12 & $7.62 * *$ & -.48 \\
\hline & Older adults & 2.71 & 0.53 & 2.57 & 2.84 & & \\
\hline \multirow[t]{2}{*}{ 4. Constructive attitudes and approaches } & Adults & 2.62 & 0.55 & 2.48 & 2.76 & 2.75 & -.30 \\
\hline & Older adults & 2.45 & 0.57 & 2.31 & 2.60 & & \\
\hline \multirow[t]{2}{*}{ 5. Self-monitoring and insight } & Adults & 3.10 & 0.49 & 2.98 & 3.23 & 0.56 & -.13 \\
\hline & Older adults & 3.04 & 0.42 & 2.94 & 3.15 & & \\
\hline \multirow[t]{2}{*}{ 6. Health service navigation } & Adults & 3.51 & 0.42 & 3.40 & 3.61 & $4.47^{*}$ & -.38 \\
\hline & Older adults & 3.32 & 0.56 & 3.18 & 3.46 & & \\
\hline \multirow[t]{2}{*}{ 7. Social integration and support } & Adults & 3.91 & 0.83 & 3.70 & 4.12 & $5.28 *$ & -.39 \\
\hline & Older adults & 3.60 & 0.73 & 3.42 & 3.78 & & \\
\hline \multirow[t]{2}{*}{ 8. Emotional distress } & Adults & 2.01 & 0.68 & 1.8 & 2.18 & 0.37 & 0.11 \\
\hline & Older adults & 2.09 & 0.72 & 1.9 & 2.27 & & \\
\hline
\end{tabular}

Notes: ${ }^{*}<<.01 ;{ }^{* *} p<.001$; The adults group consists of 23-64-year old participants, the older adults group consists of participants with age of 65 years or older. Abbreviation: heiQ, health education impact Questionnaire.

Bivariate correlations between scores on the heiQ and those on the MOS SF-36 subscales are provided in Table 8. Moderate positive correlations emerged between scores on the 2. Health directed activities subscale and those on the MOS SF-36 Vitality and Perceived mental health. Scores on the 4. Constructive attitudes and approaches subscale moderately and positively correlated with those on all the MOS SF-36 subscales, except for MOS SF-36 Bodily pains. Scores on the 5. Selfmonitoring and insight subscale moderately and positively correlated with those on the MOS SF-36 Physical functioning, MOS SF-36 Vitality, and MOS SF-36 Perceived mental health. Scores on the 7. Social integration and support subscale moderately and positively correlated with those on the MOS SF-36 Vitality and Perceived mental health. No correlation was found between scores on the 7. Social integration and support subscale and MOS SF-36 Social functioning. Finally, a weak correlation was found between scores on the 8. Emotional distress and MOS SF-36 Perceived mental health.

\section{Self-Management Skills as Predictors of Mental and Physical Health}

Scores on the heiQ 4. Constructive attitudes and approaches scale significantly and positively predicted scores on the MOS SF-36 physical health composite index $(\beta=.40, \mathrm{t}=$ $\left.3.79, \mathrm{R}^{2}=0.19, \mathrm{p}<.001\right)$. Scores on the heiQ 8. Emotional distress scale significantly and negatively predicted scores on the MOS SF-36 physical health composite index $(\beta=-.29, \mathrm{t}=$ $\left.2.83, \mathrm{R}^{2}=0.08, \mathrm{p}<.001\right)$. The regression model explained $27 \%$ of variance overall.

Scores on the heiQ 4. Constructive attitudes and approaches scale significantly and positively predicted scores on the MOS SF-36 mental health composite index $(\beta=.40, \mathrm{t}=$ $\left.3.79, \mathrm{R}^{2}=0.20, \mathrm{p}<.001\right)$. Scores on the heiQ 8. Emotional distress scale significantly and negatively predicted scores on 
Table 5 Comparisons on the heiQ Scale Scores Across Gender ( $N=299)$

\begin{tabular}{|c|c|c|c|c|c|c|c|}
\hline \multirow[t]{2}{*}{ heiQ Scales } & \multirow[t]{2}{*}{ Gender } & \multirow[t]{2}{*}{ Mean } & \multirow[t]{2}{*}{ SD } & \multicolumn{2}{|c|}{ 95\% Confidence Interval } & \multirow[t]{2}{*}{$\mathbf{F}_{(1,298)}$} & \multirow[t]{2}{*}{ Cohen's d } \\
\hline & & & & Lower Limit & Upper Limit & & \\
\hline \multirow[t]{2}{*}{ I. Positive and active engagement in life } & Males & 3.44 & 0.85 & 3.23 & 3.65 & 0.44 & 0.11 \\
\hline & Females & 3.53 & 0.77 & 3.34 & 3.72 & & \\
\hline \multirow[t]{2}{*}{ 2. Health directed activities } & Males & 2.58 & 0.78 & 2.38 & 2.77 & 0.29 & -.01 \\
\hline & Females & 2.50 & 0.84 & 2.30 & 2.70 & & \\
\hline \multirow[t]{2}{*}{ 3. Skill and technique acquisition } & Males & 2.83 & 0.55 & 2.69 & 2.97 & 0.07 & 0.05 \\
\hline & Females & 2.86 & 0.59 & 2.72 & 3.00 & & \\
\hline \multirow[t]{2}{*}{ 4. Constructive attitudes and approaches } & Males & 2,56 & 0.60 & 2.41 & 2.71 & 0.05 & -.04 \\
\hline & Females & 2.53 & 0.54 & 2.40 & 2.67 & & \\
\hline \multirow[t]{2}{*}{ 5. Self-monitoring and insight } & Males & 3.06 & 0.43 & 2.95 & 3.17 & 0.09 & 0.04 \\
\hline & Females & 3.08 & 0.49 & 2.96 & 3.20 & & \\
\hline \multirow[t]{2}{*}{ 6. Health service navigation } & Males & 3.43 & 0.52 & 3.30 & 3.56 & 0.13 & -.06 \\
\hline & Females & 3.40 & 0.48 & 3.28 & 3.52 & & \\
\hline \multirow[t]{2}{*}{ 7. Social integration and support } & Males & 3.85 & 0.72 & 3.67 & 4.03 & 1.56 & -.21 \\
\hline & Females & 3.68 & 0.85 & 3.47 & 3.89 & & \\
\hline \multirow[t]{2}{*}{ 8. Emotional distress } & Males & 2.04 & 0.72 & 2.80 & 3.33 & 0.01 & 0.04 \\
\hline & Females & 2.07 & 0.67 & 2.84 & 3.33 & & \\
\hline
\end{tabular}

Abbreviation: heiQ, health education impact Questionnaire.

Table 6 Bivariate Correlations Between the Scores on the heiQ Scales $(N=299)$

\begin{tabular}{|c|c|c|c|c|c|c|c|}
\hline heiQ Scales & 2. & 3. & 4. & 5. & 6. & 7. & 8. \\
\hline I. Positive and active engagement in life & $0.35 *$ & $0.55 *$ & $0.5 I^{* *}$ & $0.45^{*}$ & $0.43 *$ & $0.4 I^{*}$ & $-.20 *$ \\
\hline 2. Health directed activities & & $0.27^{*}$ & $0.19 *$ & $0.28 *$ & $0.11^{*}$ & $0.21 *$ & -.05 \\
\hline 3. Skill and technique acquisition & & & $0.54^{*}$ & $0.60^{*}$ & $0.60^{*}$ & $0.50 *$ & $-.13 *$ \\
\hline 4. Constructive attitudes and approaches & & & & $0.38 *$ & $0.47^{*}$ & $0.45^{*}$ & $-.35^{*}$ \\
\hline 5. Self-monitoring and insight & & & & & $0.59 *$ & $0.4 I^{*}$ & 0.08 \\
\hline 6. Health service navigation & & & & & & $0.49 *$ & -.04 \\
\hline 7. Social integration and support & & & & & & & $-.18^{*}$ \\
\hline 8. Emotional distress & & & & & & & I \\
\hline
\end{tabular}

Notes: ${ }^{*} \mathrm{p}<.01$; ** $\mathrm{p}<.001$.

Abbreviation: heiQ, health education impact Questionnaire.

the MOS SF-36 mental health composite index $(\beta=-.30, t=$ $\left.-2.90, \mathrm{R}^{2}=0.08, \mathrm{p}<.01\right)$. The regression model explained $28 \%$ of variance overall.

\section{Discussion}

The current study provided additional support about the psychometric properties of the heiQ in Italian adults with chronic conditions. Key findings included the evidence that a model with eight correlated dimensions showed good fit. While different estimation procedures were used across the previous studies, ${ }^{9-11,14}$ fit indices of the Italian heiQ appear all reasonably similar with the previous evidence, providing support for the claim that the heiQ has robust psychometric properties across translations. A strength of this finding is that it further confirms that the heiQ is a robust tool and can be used routinely 
Table 7 Bivariate Correlations Between Scores on heiQ and Those on MOS SF-36 Composite Indices ( $N=299)$

\begin{tabular}{|l|l|l|l|}
\hline heiQ Scales & 9. & 10. & Fisher's z \\
\hline I. Positive and active engagement in life & $0.26^{*}$ & $0.27^{*}$ & -0.19 \\
2. Health directed activities & 0.22 & $0.29^{*}$ & -1.32 \\
3. Skill and technique acquisition & 0.23 & 0.19 & 0.75 \\
4. Constructive attitudes and approaches & $0.46^{* *}$ & $0.46^{* *}$ & 0 \\
5. Self-monitoring and insight & $0.32^{* *}$ & $0.25^{*}$ & 1.34 \\
6. Health service navigation & $0.24^{*}$ & 0.20 & 0.75 \\
7. Social integration and support & $0.25^{*}$ & $0.26^{*}$ & -0.19 \\
8. Emotional distress & $-.38^{* *}$ & $-.39 * *$ & -0.19 \\
9. MOS SF-36 physical health composite index & 1 & $0.91^{* *}$ & \\
10. MOS SF-36 mental health composite index & $0.91^{* *}$ & 1 & \\
\hline
\end{tabular}

Notes: ${ }^{* *} \mathrm{p}<.001 ;{ }^{*} \mathrm{p}<.05$.

Abbreviations: heiQ, health education impact Questionnaire; MOS SF-36, Medical Outcomes Survey Short Form-36.

also in the Italian healthcare contexts. In addition, evidence of good fit of the questionnaire is important in Italy, like in other European countries, where the relationship between the medical staff and the patients is still asymmetrical, and there is a need of tools with robust properties capable to assess self-management skills. The inclusion of covariances between the residuals of some items improved the fit of the eight-correlated factor model. Covariances between within-scale residuals were added between the item 15 ("I feel like I am actively involved in life") and the item 8 ("I am doing interesting things in my life"), both loading on the 1 . Positive and active engagement in life dimension. This improved result could be explained by the fact that the content of the two items is largely overlapping, since in the Italian language the translation of the statement "To be actively involved" is very similar to the translation of that one "To do interesting things". Covariances were added also between the residuals of the item 32 ("I confidently give healthcare professionals the information") loading on the 6. Health service navigation and those of the item 16 ("When I have health problems, I have a clear understanding") loading on the 5. Self-monitoring and insight. It could be that for Italian patients, giving healthcare professionals information implies also having a clear understanding of their disease and symptoms, and maybe this overlapping between these two concepts could explain such result in the Italian version of the heiQ. In addition, covariances were added also between the residuals of the item 3 ("As well as seeing my doctor, I regularly monitor changes in my health") and those of the item 17 ("I carefully watch my health and do what is necessary to keep healthy"), which both loaded on the 5 . Self-monitoring and insight. Probably, this overlap could be attributed to the presence of a very similar concept in the Italian translation of the items (ie, the concept of checking health/changes in health). The two verbs "to monitor" and "to watch" can be translated in Italian with the same verb, since in Italian there is no distinction between the two verbs. This result was consistent with the finding reported in the German validation study, ${ }^{14}$ where

Table 8 Bivariate Correlations Between Scores on the heiQ Scales and Those on the MOS SF-36 Subscales (N=299)

\begin{tabular}{|c|c|c|c|c|c|c|c|c|}
\hline heiQ Scales & 9. & 10. & I I. & 12. & 13. & 14. & 15. & 16. \\
\hline I. Positive and active engagement in life & $0.24 *$ & 0.18 & 0.10 & $0.30 *$ & $0.28^{*}$ & $0.23 *$ & 0.11 & $0.30 *$ \\
\hline 2. Health directed activities & $0.26 *$ & 0.18 & -.01 & 0.16 & $0.32^{* *}$ & 0.16 & 0.20 & $0.37^{* *}$ \\
\hline 3. Skill and technique acquisition & 0.23 & 0.20 & 0.09 & 0.21 & 0.20 & 0.06 & 0.14 & 0.17 \\
\hline 4. Constructive attitudes and approaches & $0.45^{* *}$ & $0.38 * *$ & $0.24 *$ & $0.39 * *$ & $0.42 * *$ & $0.4 I^{* *}$ & $0.31 * *$ & $0.43 * *$ \\
\hline 5. Self-monitoring and insight & $0.33^{* *}$ & 0.21 & $0.24 *$ & 0.20 & $0.35^{* *}$ & 0.12 & 0.10 & $0.36 * *$ \\
\hline 6. Health service navigation & 0.14 & 0.20 & 0.22 & 0.14 & $0.30 *$ & 0.17 & 0.07 & 0.23 \\
\hline 7. Social integration and support & $0.25^{*}$ & 0.10 & 0.12 & $0.27^{*}$ & $0.34 * *$ & 0.11 & 0.12 & $0.36 * *$ \\
\hline 8. Emotional distress & $-.40 * *$ & -.23 & $-.27 *$ & $-.42 * *$ & $-.25^{*}$ & $-.40 * *$ & $-.25^{*}$ & $-.32 * *$ \\
\hline 9. MOS SF-36 Physical functioning & & $0.74 * *$ & $0.56 * *$ & $0.62 * *$ & $0.57^{* *}$ & $0.57^{* *}$ & $0.72 * *$ & $0.59 * *$ \\
\hline 10. MOS SF-36 Role limitations due to physical problems & & & $0.58 * *$ & $0.56 * *$ & $0.57^{* *}$ & $0.63 * *$ & $0.7 \mid * *$ & $0.49 * *$ \\
\hline II. MOS SF-36 Bodily pains & & & & $0.47^{* *}$ & $0.54 * *$ & $0.65^{* *}$ & $0.54 * *$ & $0.43 * *$ \\
\hline 12. MOS SF-36 General health perceptions & & & & & $0.60 * *$ & $0.59 * *$ & $0.44^{* *}$ & $0.51 * *$ \\
\hline 13. MOS SF-36 Vitality & & & & & & $0.67^{* *}$ & $0.59 * *$ & $0.85 * *$ \\
\hline 14. MOS SF-36 Social functioning & & & & & & & $0.64 * *$ & $0.6 \mathrm{I} * *$ \\
\hline 15. MOS SF-36 Role limitations due to emotional problems & & & & & & & & $0.52 * *$ \\
\hline 16. MOS SF-36 Perceived mental health & & & & & & & & I \\
\hline
\end{tabular}

Notes: ${ }^{* *} \mathrm{p}<.001 ;{ }^{*} \mathrm{p}<.05$

Abbreviations: heiQ, health education impact Questionnaire; MOS SF-36, Medical Outcomes Survey Short Form-36. 
the inclusion of the covariances between these two items improved the model, as well. Covariances were added also between the residuals of the item 3 and those of the item 37 ("I get enough chances to talk about my health"). Finally, covariances were added between the residuals of the item 34 ("My health problems do not ruin my life") and those of the item 39 ("I do not let my health problems control my life"), which both loaded on the 4 . Constructive attitudes and approaches. It could be hypothesized that in the Italian translation the meaning of the term "problems" can determine the overlapping of the content in the two items.

Reliability, assessed as internal consistency, was acceptable for 1. Positive and active engagement in life, 4. Constructive attitudes and approaches, 5. Self-monitoring and insight, 3. Skill and technique acquisition, and 6. Health service navigation subscales. It was good for 2 . Health directed activities, 8. Emotional distress, and 7. Social integration and support subscales. Overall, values of reliability were consistent with those reported in all the validation studies, conducted in Australia, Germany, Norway, The Netherlands, and France, as indicated by Table 3 .

Results of group differences showed that older adults reported moderately weaker self-management skills than adults, specifically 3. Skill and technique acquisition, 7. Social integration and support and 6. Health service navigation. This outcome highlights that health education programs for older adults should be specifically focused on the improvement of these skills in Italy. In addition, older adults with chronic conditions could be identified as a subgroup of patients needing a closer monitoring on such skills. This result appeared consistent with some data reported in the international literature, which showed that older adults with chronic diseases are a more vulnerable cohort with low adherence to medications and scarce health literacy. ${ }^{28,29}$ However, it should be noted that the current analysis did not control for some variables which could bias this difference, such as the types of chronic conditions, the number of polypathologies and polypharmacy. Another implication of the current result was that in the Italian healthcare context, the normative data of the heiQ should be adapted accounting for the age cohort; thus, it could be assumed that different norms should be calculated for adults and for older adult patients. No significant difference was observed between males and females on all the self-management skills. This outcome appeared consistent with previous research conducted on self-care behaviours of patients with chronic diseases, including self-care confidence, disease management, depression, social support and perceived control, which showed that the levels of such behaviours did not vary across gender. $^{30,31}$

Consistent with the study hypotheses and previous evidence, ${ }^{14}$ to support convergent and divergent validity, moderate associations emerged between Positive and active engagement in life, Constructive attitudes and approaches, and Social integration and support, moderate associations between Skill and technique acquisition and Health service navigation were found, and weak associations between Emotional distress and all the other self-management skills emerged.

Subsequently, the relationship between self-management skills and different QoL dimensions was examined. Bivariate correlation analyses showed that, on one hand, stronger skills of positive and active engagement in life, social integration and support and health service navigation were weakly associated with better general physical health, measured by the MOS SF-36 Physical Health composite index; on the other hand, stronger skills of self-monitoring and insight were moderately associated with better general physical health. More severe emotional distress was associated moderately with lower general physical health.

Stronger 1. Health directed activities, 5. Self-monitoring and insight and 7. Social integration and support were associated weakly with better mental health. Better constructive attitudes and approaches were associated moderately with better mental health. More severe emotional distress was associated moderately with lower general mental health. Overall, there was not a significant difference in the magnitude of the bivariate correlations between the self-management skills and the physical and mental health composite indices. This could be attributed to the fact that there was a strong correlation between the scores on the MOS SF-36 physical and mental health composite indices.

Subsequently, to test the contribution of each selfmanagement skill on the composite heath indices, a regression model was conducted including scores on all the heiQ scales as predictors and scores on the MOS SF-36 physical health and mental health composite indices as outcomes. This was an original point in the current study, as compared with previous research on the heiQ. Results indicated that higher emotional distress was the only one subscale of the heiQ associated with lower general physical QoL. This finding appeared consistent with the results reported in the German study of the heiQ ${ }^{14}$ where scores on the Emotional distress subscale were moderately and negatively associated with those on both the SF-36 Physical 
functioning and General health subscales. Overall, the current outcome suggests that emotional distress is the heiQ dimension more closely involved in physical QoL. Education programs in the Italian context should consider this result; thus, healthcare strategies aimed to improve physical health should focus first on the improvement of emotional distress. Interventions targeting depression, stress responses, helplessness, and anxiety feelings could be included in health education programs for chronic conditions, such as behavioural activation, problem-solving and cognitive therapy techniques.

Some associations between some of the heiQ subscales and some of the MOS SF-36 subscales supported criterion validity of the measure. In particular, it was confirmed by the moderate correlations between scores on the constructive attitudes and approaches subscale and those on the MOS SF-36 vitality and perceived mental health and by the moderate correlations between scores on the health directed activities subscales and those on the MOS SF-36 vitality. However, criterion validity was not supported by the absence of correlation between scores on the social integration and support subscale and those on the MOS SF-36 social functioning and by the almost weak correlation between emotional distress and perceived mental health.

In addition, in the regression analysis, stronger constructive attitudes and approaches were associated with higher mental health, while more severe emotional distress was related to lower general mental health. Indeed, higher scores on the heiQ Emotional distress subscale were moderately and negatively associated with those on the MOS SF-36 Mental health subscale also in the study performed by Schuler and colleagues. ${ }^{14}$ The evidence that emotional distress is associated with mental health is consistent with the study of Musekamp and colleagues, ${ }^{27}$ conducted on a large group of patients with chronic diseases who had undergone a disease-specific self-management group program as part of their inpatient rehabilitation. In this study, changes in self-reported self-management skills after rehabilitation predicted changes in both QoL (at a mental and physical level) and depressive symptoms at the end of rehabilitation and the 3 months follow-up. ${ }^{27}$

Overall, these findings suggested that higher emotional distress was a predictor of physical and mental QoL, while mental health was also associated with stronger constructive attitudes and approaches. The evidence that all the other selfmanagement skills did not predict either physical or mental QoL could suggest that the self-management model of chronic diseases is still not sufficiently developed in the Italian healthcare context, where patients with chronic illness are passive recipients of medical instructions. It could be hypothesized that Italian patients do not rely on selfmanagement of health to improve their QoL and rely almost completely on the staff skills and techniques.

\section{Conclusions}

Some limitations should be pointed out. A first point regards the heterogeneity of the total group, which included patients with different chronic diseases. Future research should provide additional evidence about the factor invariance of the measure across different types of diseases. Moreover, some chronic diseases were under-represented or other chronic conditions characterized by poor QoL even were not included. ${ }^{32-34}$ In addition, despite acceptable fit was reached for the CFI and the TLI indices, as their values were equal to or higher than 0.90 , good fit would require values equal to or higher than 0.95 . Reliability indices were only acceptable for the Self-monitoring and insight and Skill and technique acquisition dimensions.

Findings of the bivariate correlation analysis indicated only weak to moderate associations between the selfmanagement skills assessed by the heiQ and physical and mental QoL dimensions. This outcome could suggest further research on the psychometric properties of the heiQ, including the use of additional measures to assess criterion validity. For example, further studies could use the Psychological WellBeing Scale (PWBS). ${ }^{35}$ It covers other domains which could be related to self-management skills, such as Environmental Mastery, Purpose in Life, Autonomy, and Positive Relations.

Findings of the regression analysis showed that only emotional distress was a significant predictor of both physical and mental QoL (other things being equal) and that constructive attitudes and approaches predicted mental QoL. However, this analysis did not account for other variables, potentially associated to self-management skills, such as gender, age, illness duration, and worry/ rumination. ${ }^{36}$ Future research should examine the role of these variables in the model. Another aspect which was not assessed was the responsiveness of the questionnaire in detecting the change in self-management skills after an education program.

In conclusion, the current study demonstrated that the heiQ has robust psychometric properties and appears suitable for assessment of self-management skills in adults and older adults with chronic conditions also in Italian 
healthcare settings. The self-management skills are associated with both physical and mental dimensions of QoL.

\section{Data Sharing Statement}

The datasets during and/or analysed during the current study are available from the corresponding author on reasonable request.

\section{Ethics Approval and Consent to Participate}

All the participants provided written informed consent to participate in the study after having received a description of the aims. The study was approved by the ethical committee of the Santa Maria alle Scotte Hospital of Siena.

\section{Author Contributions}

All authors made substantial contributions to conception and design, acquisition of data, or analysis and interpretation of data; took part in drafting the article or revising it critically for important intellectual content; gave final approval of the version to be published; and agree to be accountable for all aspects of the work.

\section{Funding}

The research did not receive any type of source of funding.

\section{Disclosure}

The authors declare that they have no competing interests.

\section{References}

1. Leadley RM, Armstrong N, Lee YC, Allen A, Kleijnen J. Chronic diseases in the European Union: the prevalence and health cost implications of chronic pain. $J$ Pain Palliat Care Pharmacother. 2012;26:310-325. doi:10.3109/15360288.2012.736933

2. Lee I-M, Shiroma EJ, Lobelo F, Puska P, Blair SN, Katzmarzyk PT, for the Lancet Physical Activity Series Working Group. Effect of physical inactivity on major non-communicable diseases worldwide: an analysis of burden of disease and life expectancy. Lancet. 2012;380:219-229. doi:10.1016/S0140-6736(12)61031-9

3. Ferretti F, Pozza A, Pallassini M, et al. Gender invariance of dignity in non-terminal elderly patients with chronic diseases: a multicentric study. Qual Quant. 2019;53:1645-1656.

4. Coluccia A, Ferretti F, Fagiolini A, Pozza A. Incidence and risk factors for psychosis among migrants in Europe: a meta-analysis of cross-sectional case-control studies. Ital J Criminol. 2015;9:29-39.

5. WHO. Global Status Report on Non-Communicable Diseases 2010. Geneva: World Health Organization; 2011.

6. Cioffi R, Coluccia A, Ferretti F, Lorini F, Saggino A, Furnham A. A psychometric study of the quality perception questionnaire. Swiss $J$ Psychol. 2012;71:101-106. doi:10.1024/1421-0185/a000076

7. Fortin M, Chouinard MC, Bouhali T, Dubois MF, Gagnon C, Belanger M. Evaluating the integration of chronic disease prevention and management services into primary health care. BMC Health Serv Res. 2013;13:132. doi:10.1186/1472-6963-13-132
8. Wagner EH, Bennett SM, Austin BT, Greene SM, Schaefer JK, Vonkorff M. Finding common ground: patient-centeredness and evidence-based chronic illness care. J Altern Complement Med. 2005;11(Suppl 1):7-15. doi:10.1089/acm.2005.11.s-7

9. Osborne RH, Elsworth GR, Whitfield K. The Health Education Impact Questionnaire (heiQ): an outcomes and evaluation measure for patient education and self-management interventions for people with chronic conditions. Patient Educ Couns. 2007;66:192. doi:10.1016/j.pec.2006.12.002

10. Elsworth GR, Nolte S, Osborne RH. Factor structure and measurement invariance of the health education impact questionnaire: does the subjectivity of the response perspective threaten the contextual validity of inferences? Sage Open Med. 2015;3:2050312115585041. doi: $10.1177 / 2050312115585041$

11. Ammerlaan JW, van Os-medendorp H, Sont JK, Elsworth GR, Osborne RH. Validation of the Dutch version of the health education impact questionnaire (HEIQ) and comparison of the Dutch translation with the English, German and French HEIQ. Health Qual Life Outcomes. 2017;15:28. doi:10.1186/s12955-017-0601-4

12. Bélanger A, Hudon C, Amirall J, Fortin M, Chouinard MC, Bouhali T. Validation of a French-language version of the health education impact Questionnaire (heiQ) among chronic disease patients seen in primary care: a cross-sectional study. Health Qual Life Outcomes. 2015;13:64. doi:10.1186/s12955-015-0254-0

13. Morita R, Arakida M, Osborne RH, Nolte S, Elsworth GR, Mikami H. Adaptation and validation of the Japanese version of the Health Education Impact Questionnaire (heiQ-J) for the evaluation of self-management education interventions. Jpn $J$ Nurs Sci. 2013;10:255-266. doi:10.1111/j.1742-7924.2012.00224.x

14. Schuler M, Musekamp G, Faller H, et al. Assessment of proximal outcomes of self-management programs: translation and psychometric evaluation of a German version of the Health Education Impact Questionnaire (heiQ). Qual Life Res. 2013;22:1391-1403. doi:10.1007/s11136-012-0268-6

15. Wahl AK, Osborne RH, Langeland E, et al. Making robust decisions about the impact of health education programs: psychometric evaluation of the Health Education Impact Questionnaire (heiQ) in diverse patient groups in Norway. Patient Educ Couns. 2016;99:1733-1738. doi:10.1016/j.pec.2016.05.001

16. Ware JE, Sherbourne CD. The MOS 36-item short-form health survey (SF-36): I. Conceptual framework and item selection. Med Care. 1992;30:473-483. doi:10.1097/00005650-199206000-00002

17. American Educational Research Association. American Psychological Association \& National Council of Measurement in Education. Standards for Educational and Psychological Testing. 2014.

18. Coluccia A, Ferretti F, Fagiolini A, Pozza A. Psychometric properties and relations with coping and family strain of the Health Services and Caregiver Experience questionnaire (HSCE): an outcome measure of informal caregivers' experience for inpatient care in Italy. $B M C$ Health Serv Res. 2017;17:494. doi:10.1186/s12913-017-2317-x

19. Hawkins M, Osborne RH. Questionnaire translation and cultural adaption procedure version 1.0. 2010.

20. American Psychological Association. Ethical principles of psychologists and code of conduct. Am Psychol. 2002;57:1060-1073. doi:10.1037/0003-066X.57.12.1060

21. Apolone G, Mosconi P. The Italian SF-36 health survey: translation, validation and norming. $J$ Clin Epidemiol. 1998;51:1025-1036. doi:10.1016/S0895-4356(98)00094-8

22. Bollen KA. A new incremental fit index for general structural equation models. Social Methods Res. 1989;17:303-316. doi:10.1177/ 0049124189017003004

23. Hu LT, Bentler PM. Cutoff criteria for fit indexes in covariance structure analysis: conventional criteria versus new alternatives. Struct Equ Modeling. 1999;6:1-55. doi:10.1080/10705519909540118 
24. Bentler PM, Bonett DG. Significance tests and goodness of fit in the analysis of covariance structures. Psychol Bull. 1980;88:588. doi:10.1037/0033-2909.88.3.588

25. Nunnally JC, Bernstein IH. Psychometric Theory. 3rd ed. New York: McGraw Hill; 1994.

26. Cohen J, Cohen P, West SG, Aiken LS. Applied Multiple Regression/ Correlation Analysis for the Behavioral Sciences. New York: Routledge; 1998.

27. Musekamp G, Bengel J, Schuler M, Faller H. Improved self-management skills predict improvements in quality of life and depression in patients with chronic disorders. Patient Educ Couns. 2016;99:1355-1361. doi:10.1016/j.pec.2016.03.022

28. Federman AD, Wolf MS, Sofianou A, et al. Self-management behaviors in older adults with asthma: associations with health literacy. J Am Geriatr Soc. 2014;62:872-879. doi:10.1111/jgs.12797

29. Carolien G, Sino M, Sietzema M, Egberts G, Schuurmans MJ. Medication management capacity in relation to cognition and self-management skills in older people on polypharmacy. $J \mathrm{Nutr}$ Health Aging. 2014;18:44.

30. Kueh YC, Morris T, Borkoles E, Shee H. Modelling of diabetes knowledge, attitudes, self-management, and quality of life: a cross-sectional study with an Australian sample. Health Qual Life Outcomes. 2015;13:129. doi:10.1186/s12955-015-0303-8

31. Heo S, Moser DK, Lennie TA, Riegel B, Chung ML. Gender differences in and factors related to self-care behaviors: a cross-sectional, correlational study of patients with heart failure. Int J Nurs Stud. 2008;45:1807-1815. doi:10.1016/j.ijnurstu.2008.05.008
32. Coluccia A, Pozza A, Gusinu R, Gualtieri G, Muzii VF, Ferretti F. Do patients with chronic low-back pain experience a loss of health-related quality of life? A protocol for a systematic review and meta-analysis. BMJ Open. 2020;10(2):e033396. doi:10.1136/ bmjopen-2019-033396

33. Ferretti F, Coluccia A, Gusinu R, Gualtieri G, Muzii VF, Pozza A. Quality of life and objective functional impairment in lumbar spinal stenosis: a protocol for a systematic review and meta-analysis of moderators. BMJ Open. 2019;9(11):e032314. doi:10.1136/bmjopen2019-032314

34. Pozza A, Ferretti F, Coluccia A. Perceived physical health in obsessive-compulsive disorder: a protocol for a systematic review and meta-analysis. BMJ Open. 2019;9(6):e026261. doi:10.1136/ bmjopen-2018-026261

35. Ryff CD. Happiness is everything, or is it? Explorations on the meaning of psychological well-being. J Pers Soc Psychol. 1989;57:1069-1081. doi:10.1037/0022-3514.57.6.1069

36. Barcaccia B, Baiocco R, Pozza A, Pallini S, Mancini F, Salvati M. The more you judge the worse you feel. A judgemental attitude towards one's inner experience predicts depression and anxiety. Pers Individ Diff. 2019;138:33-39. doi:10.1016/j.paid.2018.09.012
Psychology Research and Behavior Management

\section{Publish your work in this journal}

Psychology Research and Behavior Management is an international, peer-reviewed, open access journal focusing on the science of psychology and its application in behavior management to develop improved outcomes in the clinical, educational, sports and business arenas Specific topics covered in the journal include: Neuroscience, memory and decision making; Behavior modification and management; Clinica applications; Business and sports performance management; Social and developmental studies; Animal studies. The manuscript management system is completely online and includes a very quick and fair peer-review system, which is all easy to use. Visit http://www. dovepress.com/testimonials.php to read real quotes from published authors. 